\title{
Animating Character Images in 3D Space
}

\author{
Bing-Yu Chen* \\ National Taiwan University \\ Shih-Chiang Dai ${ }^{\dagger}$ \\ National Taiwan University
}
Shuen-Huei Guan
Digimax

Tomoyuki Nishita

The University of Tokyo
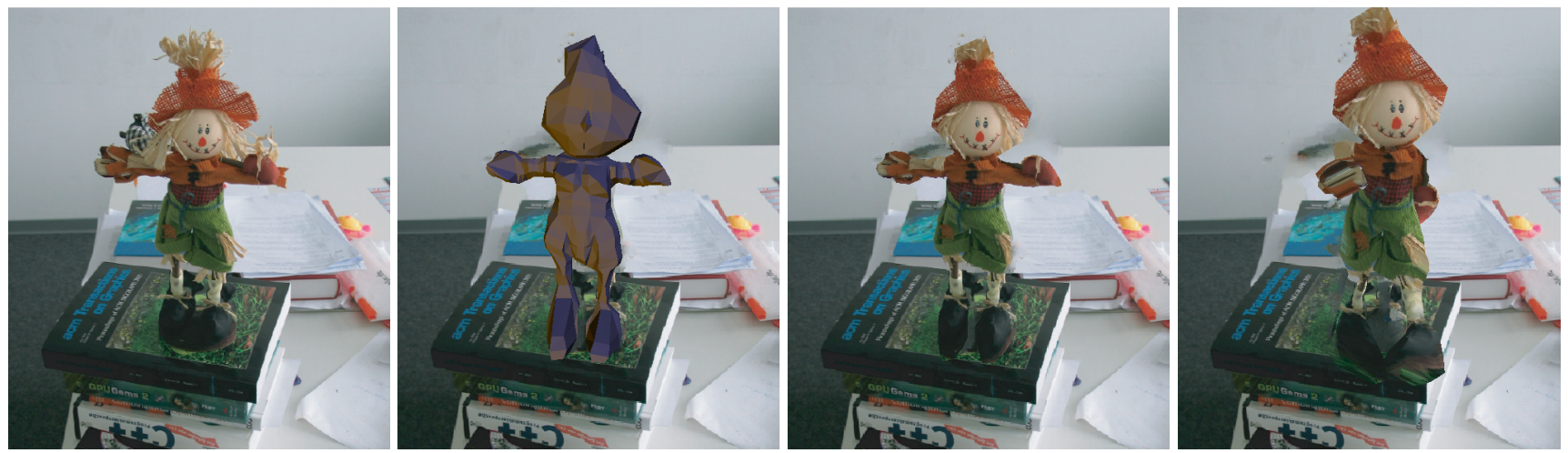

Figure 1: From left to right: the original input image; the template character model after fitting to the character image; the character model textured by the character image; the motion data is applied to animate the character model.

\section{Introduction}

In this extended abstract, we present a system that allows the user to animate character images in 3D space by applying an existed 3D character model with motion data. The character model with skeleton rigged is used as a template model to fit the silhouette of the character image. After assigning some corresponding points between the character image and template model, the system then fits the model to the image and transfer the colors and patterns of the image to the model as the textures. Finally, the user can apply any motion data to animate the fitted 3D character model in 3D space.

\section{Overview}

The input of our system is an image and a skeleton rigged 3D character model and a 2D character image. The system first samples some points on the contour of the character image as the vertices in $3 \mathrm{D}$ space, and the points form a close loop as a "contour loop" $C$. Then, the system roughly generates silhouette vertices from the 3D character model, which also form a close loop as a "silhouette loop" $S$. Then, our model fitting algorithm is applied to fit $S$ to $C$ while considering the original shape of the 3D character model. Finally, we can apply any motion data to the $3 \mathrm{D}$ character model or add any visual effect in $3 \mathrm{D}$ space.

\section{Model Fitting Algorithm}

Given a contour loop $C$ extracted from the input character image $I$ and a silhouette loop $S=\left\{s_{i} \mid i=1, \cdots, n\right\}, s_{n}=s_{1}$ with $n$ silhouette vertices of the 3D character model $T$, we have to match $S$ with $C$. The user first drag $m \leq n$ vertices of the silhouette loop $S^{\prime}=\left\{s_{p(j)} \mid j=1, \cdots, m\right\} \subseteq S, s_{p(m)}=s_{p(1)}=s_{1}$ to their corresponding points of $C$ manually, where $p(j)=i$ denotes an index mapping from the dragged vertices $s_{p(j)}$ to the silhouette vertices $s_{i}$, and $s_{p(1)}=s_{1}$. Then, the system fits the remaining $n-m$ silhouette vertices to $C$ automatically while satisfying the constraint: $\overline{s_{i} s_{i+1}}=\overline{s_{p(j)} s_{p(j+1)}} /(p(j+1)-p(j)), p(j) \leq i \leq p(j+1)$.

After fitting $S$ to $C$, we have to deform the shape of $T$ to match that of $I$ while satisfying the constraint (i.e., $S^{\prime}$ ). We can first keep the

\footnotetext{
*e-mail: robin@ntu.edu.tw

†e-mail: jeffrey@cmlab.csie.ntu.edu.tw

†e-mail: drake.guan@gmail.com

$\S$ e-mail: nis@is.s.u-tokyo.ac.jp
}

$z$ coordinate fixed and only consider the 3D model $T$ as a 2D mesh $T_{x y}$. Then, as-rigid-as-possible shape manipulation [Igarashi et al. 2005] is used to deforme $T_{x y}$ with constrained $S^{\prime}$. Through a sparse linear solver, we can fit the remaining vertices of $T_{x y}$ within a sec. After fitting the skin (only $x-y$ coordinates) of $T$, we still have to fit the original skeleton of $T$ corresponding to the fitted skin.

Before skeleton fitting, we first project $T$ and its skeleton joints onto $x-y$ plane (i.e., $T_{x y}$ ), and record each joint position by barycentric coordinate of the triangle which contains the joint. If there exists several triangles contain the same joint, we choose the one nearest to the joint in the original 3D space and belongs to that bone.

After fitting the skin and skeleton, we have transformed $T$ to fit $I$ by adjusting its projected $x-y$ coordinates in $2 \mathrm{D}$ space (i.e., $T_{x y}$ ). However, the quantity of the third (z) coordinate (or thickness) must also be revised to generate a convincing fitted 3D character model. In our experience, we observed that the distance between the bone and the skin is highly correlated to the average distance between the silhouette vertices $s_{i} \in S$ and the bone $b_{k} \in B$ they belong to. Hence, for each $b_{k}$ we record the average distance $d_{k}$ to its nearest silhouette loop $S$ before the model fitting. If the vertex $s_{i}$ belongs to several bones, we compute the average with its bone weight $\omega_{i k}$ as $d_{k}=\sum_{i}^{n-1} \omega_{i k} d_{i k} /(n-1), d_{i k}=\left\|s_{i}-b_{k}\right\|$, where $n$ is the number of $s_{i} \in S$ and $\omega_{i k}=0$ if $s_{i}$ and $b_{k}$ have no binding relationship. After skin fitting, we can then compute the new average distance $d_{k}^{\prime}$ by using the new position of $s_{i}$. Then, the new $z$ value of each vertex is scaled by the ratio: $\sum_{k} \omega_{v k}\left(d_{k}^{\prime} / d_{k}\right)$, where $\omega_{v k}$ is the binding weight between the vertex $v \in T$ and the bone $b_{k}$.

\section{Result}

Our system is implemented in C++ with OpenGL, and the character deformation is performed by following the standard linear blend skinning (LBS) method. The motion data is downloaded from http://mocap.cs.cmu.edu/. Fig. 1 shows the result. The computation time is interactive except inpainting and silhouette cut out. The user interaction time is about $10 \mathrm{~min}$. for a trained user.

\section{References}

Igarashi, T., Moscovich, T., And Hughes, J. F. 2005. Asrigid-as-possible shape manipulation. ACM Trans. Gr. 24, 3, 1134-1141. (SIGGRAPH 2005 Conf. Proc.). 